\title{
The Fate of Formamide in a Fragmenting Protoplanetary Disk
}

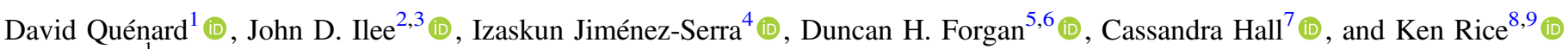 \\ ${ }^{1}$ School of Physics and Astronomy, Queen Mary University of London, Mile End Road, London E1 4NS, UK; j.d.ilee@leeds.ac.uk \\ ${ }^{2}$ School of Physics \& Astronomy, University of Leeds, Leeds LS2 9JT, UK \\ ${ }^{3}$ Institute of Astronomy, University of Cambridge, Madingley Road, Cambridge CB3 0HA, UK \\ ${ }^{4}$ Centro de Astrobiología (CSIC/INTA), Ctra. de Torrejón a Ajalvir km 4, E-28850, Torrejón de Ardoz, Spain \\ ${ }^{5}$ SUPA, School of Physics and Astronomy, University of St. Andrews, North Haugh, St. Andrews KY16 9SS, UK \\ ${ }^{6}$ St. Andrews Centre for Exoplanet Science, University of St. Andrews, St. Andrews KY16 9SS, UK \\ ${ }^{7}$ Department of Physics and Astronomy, University of Leicester, Leicester LE1 7RH, UK \\ ${ }^{8}$ SUPA, Institute for Astronomy, University of Edinburgh, Blackford Hill, Edinburgh EH9 3HJ, UK \\ ${ }^{9}$ Centre for Exoplanet Science, University of Edinburgh, Edinburgh EH9 3HJ, UK \\ Received 2018 May 11; revised 2018 September 11; accepted 2018 September 25; published 2018 November 13
}

\begin{abstract}
Recent high-sensitivity observations carried out with the Atacama Large Millimeter Array have revealed the presence of complex organic molecules $(\mathrm{COMs})$ such as methyl cyanide $\left(\mathrm{CH}_{3} \mathrm{CN}\right)$ and methanol $\left(\mathrm{CH}_{3} \mathrm{OH}\right)$ in relatively evolved protoplanetary discs. The behavior and abundance of COMs in earlier phases of disk evolution remain unclear. Here, we combine a smoothed particle hydrodynamics simulation of a fragmenting, gravitationally unstable disk with a gas-grain chemical code. We use this to investigate the evolution of formamide $\left(\mathrm{NH}_{2} \mathrm{CHO}\right)$, a prebiotic species, in both the disk and in the fragments that form within it. Our results show that formamide remains frozen onto grains in the majority of the disks where the temperatures are $<100 \mathrm{~K}$, with a predicted solid-phase abundance that matches those observed in comets. Formamide is present in the gas phase in three fragments as a result of the high temperatures $(\geqslant 200 \mathrm{~K})$, but remains in the solid phase in one colder $(\leqslant 150 \mathrm{~K})$ fragment. The timescale over which this occurs is comparable to the dust sedimentation timescales, suggesting that any rocky core that is formed would inherit their formamide content directly from the protosolar nebula.
\end{abstract}

Key words: astrochemistry - hydrodynamics - planets and satellites: formation - protoplanetary disks

\section{Introduction}

The origin of the prebiotic content at the surface of the primitive Earth remains unclear. It is postulated that a large quantity of prebiotic material might have been brought to Earth by comets and meteorites during its early formation (Caselli et al. 2012). This hypothesis is supported by the detection of numerous prebiotically relevant molecular species in comets such as Hale-Bopp (Bockelée-Morvan et al. 2000), Lemmon and Lovejoy (Biver et al. 2014), and more recently 67P/ Churyumov-Gerasimenko (Goesmann et al. 2015; Altwegg et al. 2017).

Among these species is formamide $\left(\mathrm{NH}_{2} \mathrm{CHO}\right)$, an important precursor of pre-genetic and pre-metabolic compounds such as nucleic acids, nucleobases, sugars, and amino acids (see Saladino et al. 2012). The reservoir of complex organic molecules (COMs) - such as formamide-in comets is expected to derive from the previous stages of star formation. Formamide has indeed been detected in the hot envelopes around low-mass protostars (e.g., Kahane et al. 2013). The unrivalled sensitivity of the Atacama Large Millimeter Array (ALMA) has opened up the possibility to also study COM chemistry in protoplanetary disks. However, only two COMs have been detected so far in the gas phase in these objects: methyl cyanide $\left(\mathrm{CH}_{3} \mathrm{CN}\right.$, Öberg et al. 2015) and methanol $\left(\mathrm{CH}_{3} \mathrm{OH}\right.$, Walsh et al. 2016). A large proportion of these COMs are thought to remain frozen onto dust grains, making their detection in the gas phase challenging (see, e.g., Walsh et al. 2014).

There have been several theoretical studies of the evolution and inheritance of chemistry during the youngest stages of protoplanetary disks. Some have considered the chemical link between a surrounding envelope and an axisymmetric disk (e.g., Visser et al. 2011; Drozdovskaya et al. 2016; Furuya et al. 2017), finding that chemical composition depends on the route taken by material to the forming disk. Other works have chosen to concentrate on the chemistry of isolated disks, modeling the full three-dimensional hydrodynamic evolution within the disk itself (e.g., Ilee et al. 2011; Evans et al. 2015; Yoneda et al. 2016), finding that dynamically induced changes in the temperature and density of the disk material can significantly influence the chemical composition. To date, only one study has followed the time-dependent chemical evolution of a disk that forms protoplanetary fragments (Ilee et al. 2017), but this work did not consider the formation and evolution of complex molecules. Therefore, the formation and evolution of COMs from the protostellar phase to the birth of a planetary system remains unclear.

In this paper, we investigate the fate of one such COM, formamide, during one of the earliest phases of disk evolution. We consider a massive, self-gravitating protostellar disk that undergoes fragmentation, which we model using smoothed particle hydrodynamics (SPH) coupled with a gas-grain chemical evolution code. We examine the abundance of formamide in the different environments of the disk (e.g., cold outer regions, regions undergoing shocks) and particularly in the four protoplanetary fragments formed during the simulation. We show that our models broadly reproduce observations of COMs in comets, and that formamide may survive around the fragments long enough to be incorporated into protoplanets' rocky cores, implying the possibility of direct inheritance of formamide from the protosolar epoch. 


\section{Methodology}

\subsection{Hydrodynamic Modeling}

We use the same radiation-hydrodynamic disk simulation as in Ilee et al. (2017), which is also "Run 2" from Hall et al. (2017). Full details of the simulation setup are given in these works, so we only briefly reiterate key aspects here. We simulate a $0.25 M_{\odot}$ disk, with a $1 M_{\odot}$ central protostar. The gas in the disk is represented by $4 \times 10^{6} \mathrm{SPH}$ particles distributed between 10 and $100 \mathrm{au}$, with an initial surface density profile that scales as $\Sigma(r) \propto r^{-1}$ and an initial sound speed profile that scales as $c_{\mathrm{s}} \propto r^{-0.5}$. We include the modified radiation transfer scheme of Forgan et al. (2009) in which the gas is able to cool radiatively according to its local optical depth (calculated from the gravitational potential), and can exchange energy with neighboring fluid elements via fluxlimited diffusion.

Four fragments are formed during this simulation (labeled with ascending radial distance from the central star as John, Paul, George and Ringo; see Ilee et al. 2017). John and Paul are tidally disrupted at $t=3081 \mathrm{yr}$ and $t=3514 \mathrm{yr}$, respectively, while George and Ringo survive until the end of the simulation. John contains the most mass $\left(10.3 M_{\text {Jup }}\right)$ but undergoes tidal disruption, George is the most massive surviving fragment $\left(8.2 M_{\text {Jup }}\right)$, Ringo reaches $5.4 M_{\text {Jup }}$ at the end of the simulation, and Paul is the least massive fragment, reaching $3.7 M_{\text {Jup }}$ before being disrupted.

\subsection{Chemical Modeling}

The chemical calculations are performed using UCLCHEM (Viti et al. 2004; Holdship et al. 2017), a gas-grain chemical evolution code. The gas-phase reactions are taken from the UMIST database (McElroy et al. 2013), with additional reactions from Quénard et al. (2018). The grain surface reaction network is the one presented in Quénard et al. (2018). Several thermal and non-thermal processes are considered on the grain surface, such as diffusion via thermal hopping and quantum tunneling (Hasegawa et al. 1992), thermal desorption (Hasegawa et al. 1992), cosmic-ray desorption, direct-UV and secondary-UV desorptions (McElroy et al. 2013; Holdship et al. 2017), and chemically reactive desorption (Minissale et al. 2016).

The network contains 364 species (243 in the gas phase and 121 on the grain surface) and 3446 reactions. The starting elemental abundances are the same as those in Quénard et al. (2018). Binding energies of molecules are taken from Wakelam et al. (2017; see Table 1). We note that we do not consider three-body reactions, expected to be important in high-density environments, within our chemical model. However, Ilee et al. (2011) included three-body reactions in their study of the chemical composition of a massive disk, finding that they have a small effect on the molecular abundances (see their Section 3.3).

The chemical modeling is performed in four steps, where the first three initialize the chemical conditions prior to the full protoplanetary disk phase (i.e., the final abundances of phases 1,2 and 3 are used as initial abundances for phases 2, 3 and 4, respectively).

The first step (Phase 1) corresponds to the diffuse cloud phase, where we follow the chemistry of a low-density cloud $\left(n_{\mathrm{H}}=10^{2} \mathrm{~cm}^{-3}\right.$ and $A_{\mathrm{V}}=2 \mathrm{mag}$ ) over $10^{6}$ years at a kinetic temperature $T_{\text {kin }}=10 \mathrm{~K}$. Only gas-phase chemistry plays a
Table 1

Binding Energies of Formamide and Related Species Adopted in Our Chemical Model, Taken from Wakelam et al. (2017)

\begin{tabular}{lc}
\hline \hline Species & Binding Energy \\
& $E_{\mathrm{D}}(\mathrm{K})$ \\
\hline $\mathrm{CO}$ & 1300 \\
$\mathrm{CO}_{2}$ & 2600 \\
$\mathrm{CH}_{4}$ & 960 \\
$\mathrm{HCN}$ & 3700 \\
$\mathrm{H}_{2} \mathrm{O}$ & 5600 \\
$\mathrm{CH}_{3} \mathrm{OH}$ & 5000 \\
$\mathrm{NH}_{3}$ & 5500 \\
$\mathrm{NH}_{2}$ & 3200 \\
$\mathrm{H}_{2} \mathrm{CO}$ & 4500 \\
$\mathrm{NH}_{2} \mathrm{CHO}$ & 6300 \\
\hline
\end{tabular}

role at this stage since the $A_{\mathrm{V}}$ is low, and all grain surface species are either destroyed or desorbed via non-thermal processes (see the left panel of Figure 1).

The second step (Phase 2) is the pre-stellar phase, where the cloud contracts for $\sim 5.4 \times 10^{6} \mathrm{yr}$ until it reaches a final density of $n_{\mathrm{H}}=1 \times 10^{11} \mathrm{~cm}^{-3}$ following a freefall collapse parameterization (Rawlings et al. 1992; Holdship et al. 2017). The gas temperature remains at $10 \mathrm{~K}$ during this phase. The cloud remains at low $A_{\mathrm{V}} \lesssim 4$ mag with $n_{\mathrm{H}} \lesssim 2 \times 10^{3} \mathrm{~cm}^{-3}$ for the first $\sim 4.7 \times 10^{6}$ yr. During this period, the chemistry does not vary significantly and the abundances of formamide, as well as those of species such as $\mathrm{CO}, \mathrm{H}_{2} \mathrm{O}, \mathrm{H}_{2} \mathrm{CO}$, and $\mathrm{NH}_{2}$, remain relatively the same (see the left panel of Figure 1). At approximately $6.3 \times 10^{6} \mathrm{yr}$, almost all molecules experience a steep increase in gas-phase abundance. As discussed in Quénard et al. (2018), the high density allows molecular species to be efficiently produced on grain surfaces, since the high visual extinction prevents UV photons from destroying or desorbing molecules. These newly formed species are then injected into the gas phase via non-thermal desorption processes such as chemical reactive desorption. The abundances of $\mathrm{H}_{2} \mathrm{O}, \mathrm{H}_{2} \mathrm{CO}, \mathrm{NH}_{2}$, and $\mathrm{NH}_{2} \mathrm{CHO}$ drastically decrease after $6.4 \times 10^{6}$ years, as a result of the severe freezing out expected at the high-density regime in pre-stellar cores.

The third step (Phase 3) is the warming-up (or protostellar/ hot corino) phase. While the density is kept constant and the temperature increases from 10 to $300 \mathrm{~K}$ following a rate defined by Awad et al. (2010), due to the increasing grain surface temperature, this phase enables the formation of COMs via radical-radical addition. Once the dust temperature exceeds the desorption energies shown in Table 1, molecular species are thermally desorbed into the gas phase. We also emphasize that higher temperatures also help to overcome the activation barrier of the endothermic reactions present in our gas-phase chemical network, which triggers a richer gas-phase chemistry.

The fourth step (Phase 4) is the protoplanetary disk phase, when we run UCLCHEM for a subset of 100,000 particles taken from the SPH simulation. We proceed in this way to make it possible to undertake the calculation of the chemical evolution of the disk in a reasonable time frame using the large network of UCLCHEM. The subset of particles was selected randomly, but the selection was confined to within a smoothing-length of the midplane in order to preserve the radial surface density structure of the disk. The subset is large enough to ensure that all the physical regimes of the disk are probed. For each particle within this subset, the starting abundance is taken to be 

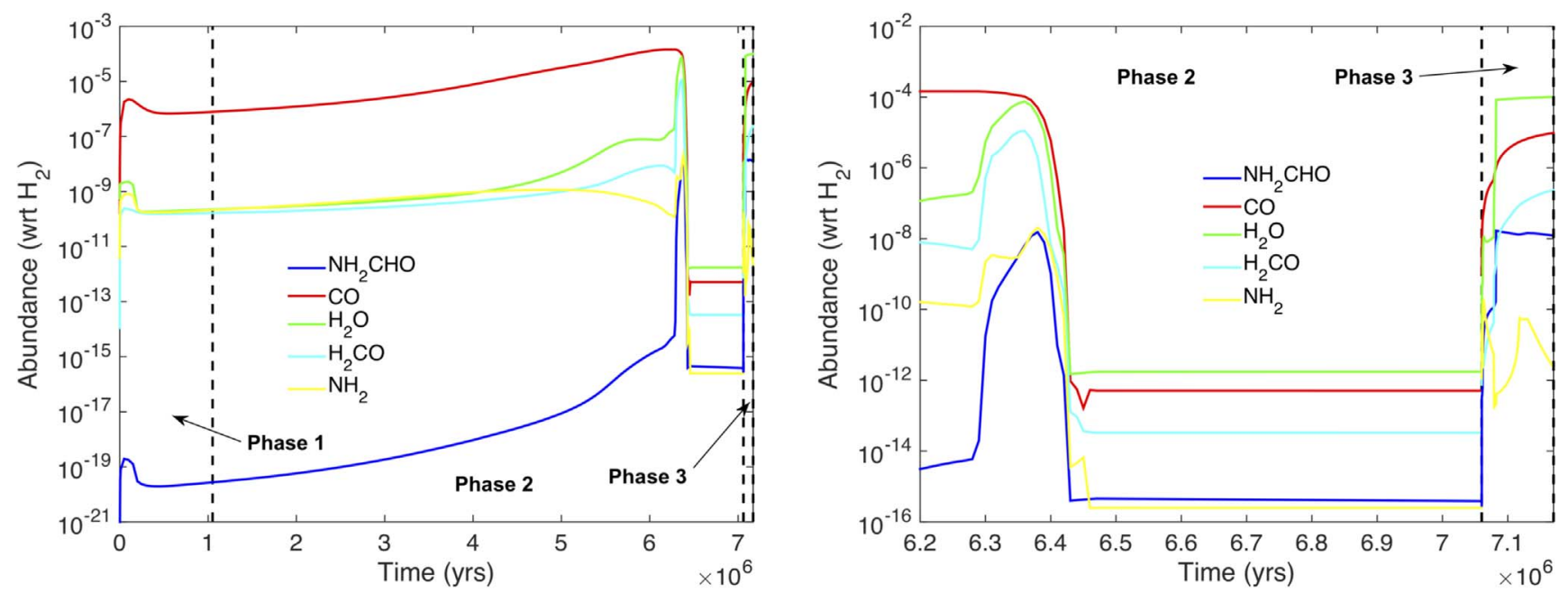

Figure 1. Gas-phase abundance of $\mathrm{NH}_{2} \mathrm{CHO}$ (formamide), $\mathrm{CO}, \mathrm{H}_{2} \mathrm{O}, \mathrm{NH}_{2}$, and $\mathrm{H}_{2} \mathrm{CO}$ across the pre-disk phases in our chemical modeling. Left panel: abundances across phase 1-3 for the full $\sim 7.2 \mathrm{Myr}$ considered. Right panel: a closeup of the final Myr showing phases $2 \& 3$.

the final abundance of the warming-up (protostellar) phase. Since the subset is drawn from close to the midplane, we set the visual extinction $\left(A_{V}\right)$ in this phase to $>100$ mag, such that any photo-processing can be considered negligible. For all phases, we assume an external radiation field of $G_{0}=1$ Habing and a standard cosmic-ray ionization rate of $\zeta=$ $1.3 \times 10^{-17} \mathrm{~s}^{-1}$.

We have benchmarked the chemical abundances obtained in our work with those calculated by Ilee et al. (2017), who used a smaller chemical network (125 species-89 in the gas, 36 on dust grains-and 1334 reactions; see their Section 2.2). We find a good agreement between the two networks, especially for molecules that are key in the production of formamide (e.g., $\mathrm{CO}, \mathrm{CO}_{2}, \mathrm{H}_{2} \mathrm{CO}, \mathrm{H}_{2} \mathrm{O}, \mathrm{NH}_{2}$, and $\mathrm{NH}_{3}$ ), with abundances differing by less than a dex. This result is reassuring given the large discrepancy in size between the two networks.

\section{Results}

\subsection{Formamide $\left(\mathrm{NH}_{2} \mathrm{CHO}\right)$ in the Disk}

Figure 2 shows the abundance of formamide in four different time snapshots in the protoplanetary disk. The first snapshot at $204 \mathrm{yr}$ shows the initial high gas-phase abundance of formamide across the disk, inherited from the protostellar (hot corino) phase. Rapidly, around $850 \mathrm{yr}$, all species are frozen out onto dust grains because of the cold temperatures of the disk $(T \lesssim 20 \mathrm{~K})$ combined with high densities $\left(n\left(\mathrm{H}_{2}\right) \gtrsim 10^{10} \mathrm{~cm}^{-3}\right)$. Later, formamide is released back into the gas phase via thermal desorption in regions where $T \gtrsim 100 \mathrm{~K}$. The magnitude of these effects depends strongly on the position of the particle within the disk (e.g., whether they reside in shocked regions, or within fragments). The total amount of formamide (gas phase plus solid phase) does not vary significantly with time, since the bulk of the formamide has already been produced during the protostellar phase. Therefore, the formamide abundance in the gas phase or solid phase is mainly driven by the temperature, leading either to freeze-out or to desorption.

In the innermost parts of the disk $(r<15 \mathrm{au})$, Figure 2 shows that formamide is in the gas phase. This is due to the high gas kinetic temperatures $(T=100-400 \mathrm{~K})$, which thermally desorb formamide from grains. Note that the presences of $\mathrm{UV}$ and/or X-ray radiation fields from the central protostar are not taken into account here. Therefore, the abundance of formamide at $r<15$ au might be lower due to the photodestruction of this molecule. At larger radii, the high visual extinction $\left(A_{V} \geqslant 470\right.$ mag $)$ associated with the large densities in the disk (up to $10^{13} \mathrm{~cm}^{-3}$ in the midplane) will shield formamide from any radiation fields (both stellar and external).

The gas-phase formamide abundance in the outermost parts of the disk typically remains lower than $10^{-12}$, even reaching $\sim 10^{-18}$ in some regions. As the temperature is also low (close to $10 \mathrm{~K}$ ), the bulk of the formamide remains frozen onto dust grains (with a constant abundance of $10^{-8}$ ) in the arms and the outer parts of the disk. In Section 4.1, we compare these solid-phase abundances of formamide in the outer disk predicted by our model with those measured observationally in solar system comets.

Figure 3 presents the evolution of the column density of the disk overlaid with contours showing the location of the midplane snowlines for $\mathrm{CO}, \mathrm{H}_{2} \mathrm{O}$, and $\mathrm{NH}_{2} \mathrm{CHO}$. The snowlines are defined by the radii at which the gas-phase column density of a species is equal to that of the column density of the corresponding ice-phase species, i.e., $N_{\text {gas }}=N_{\text {ice }}$ (see Section 4.2 of Ilee et al. 2017). The CO snow line is much larger than the one for $\mathrm{H}_{2} \mathrm{O}$ because of the larger desorption temperature for the latter. Since formamide is desorbed under similar conditions to water (their binding energies are similar, $E_{\mathrm{D}}=5600 \mathrm{~K}$ for $\mathrm{H}_{2} \mathrm{O}$ versus $E_{\mathrm{D}}=6300 \mathrm{~K}$ for $\mathrm{NH}_{2} \mathrm{CHO}$, see Table 1), their snowlines are practically the same. We note that the formamide/water snow line is only present for the hottest fragments (George and John; middle panel of Figure 3), because the amount of gas-phase formamide (or water) in Paul and Ringo is not high enough to match the amount of this molecule on the surfaces of dust grains.

\subsection{Radial Distribution of Formamide in the Fragments}

Figure 4 presents the median abundance of formamide for the four fragments as a function of distance from the fragment's center for different times. These abundances are calculated by taking into account the median of all abundance values within shells of $0.2 \mathrm{au}$ in width, with 50 shells covering a radial distance of $10 \mathrm{au}$ around each fragment). The time range for each fragment is taken between their formation time and the end of the simulation (or destruction time where appropriate, 

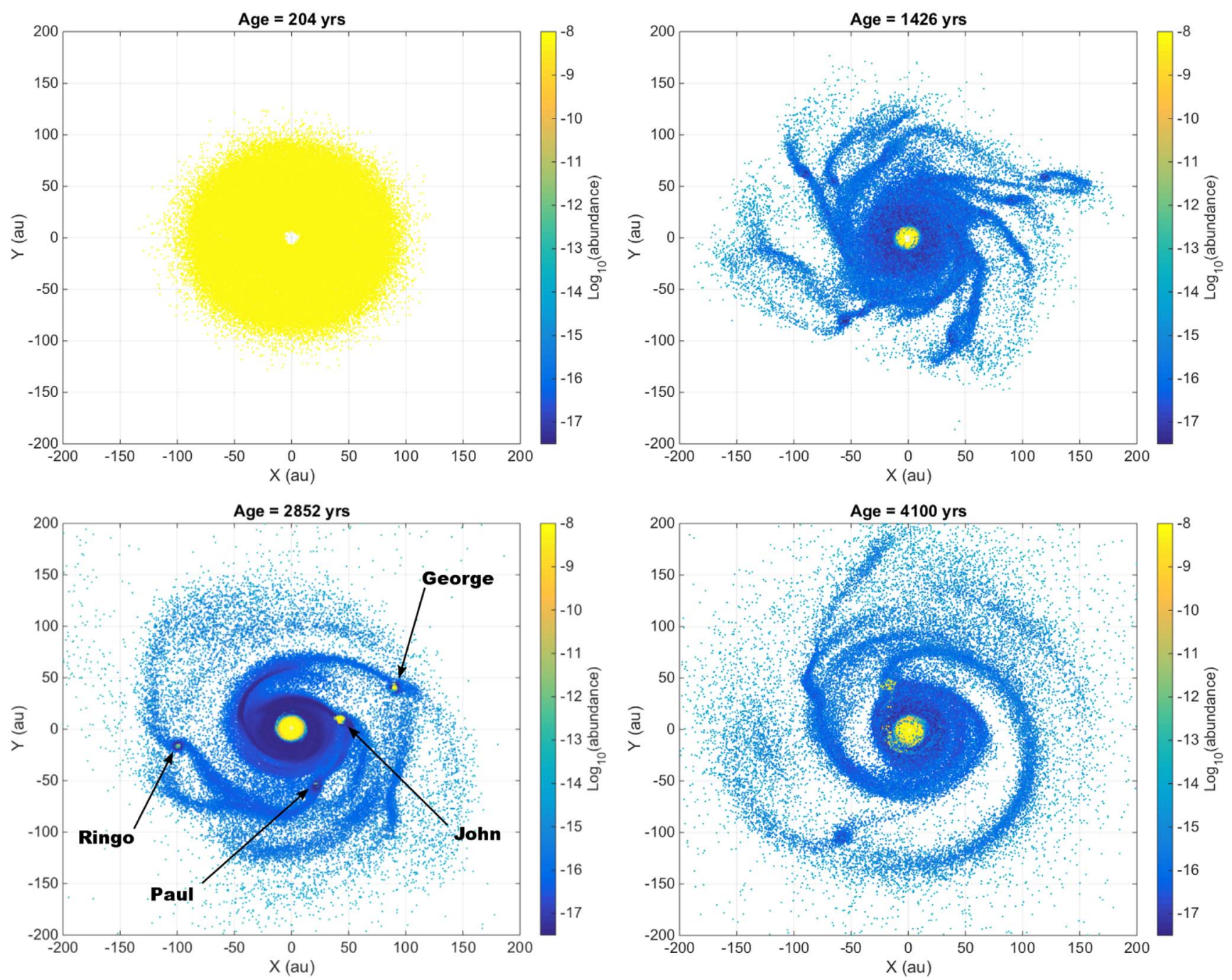

Figure 2. Gas-phase abundance of formamide across the protoplanetary disk for four different snapshots covering $4100 \mathrm{yr}$ of disk evolution. Formamide is kept on the grain surface in the majority of the disk, but with time it goes back to the gas phase in the central part of the disk and around some fragments.
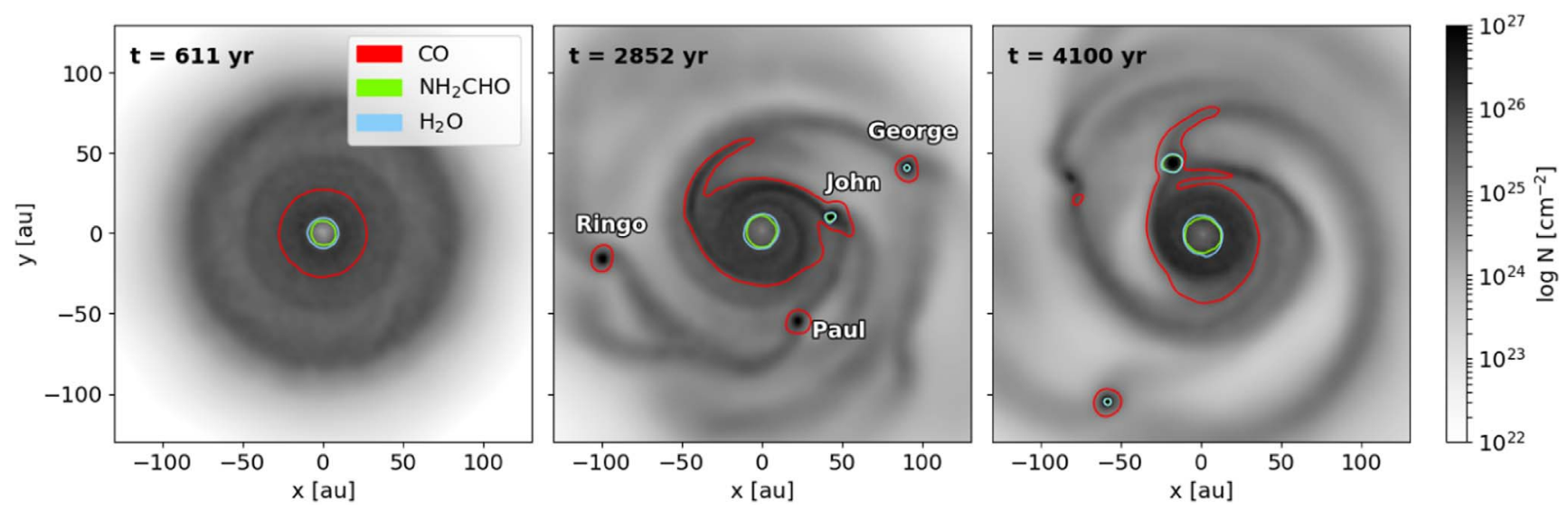

Figure 3. Evolution of the total column density of the disk (gray scale) overlaid with the snowlines of $\mathrm{CO}$ (red), $\mathrm{NH}_{2} \mathrm{CHO}$ (green), and $\mathrm{H}_{2} \mathrm{O}$ (blue). The snowlines quickly deviate from the expected concentric ring structure due to the dynamic evolution of the disk.

i.e., $2037-3081 \mathrm{yr}$ for John; $1630-3514 \mathrm{yr}$ for Paul; $2037-4100 \mathrm{yr}$ for George; and 2445-4100 yr for Ringo-see also Table 2 of Ilee et al. 2017).

For George, the radius of gas-phase formamide increases with time, to be $\sim 7$ au at the end of the simulation. This is due to the increasing temperature of the fragment (up to temperatures of $1100 \mathrm{~K}$ ), caused by its inward migration and increasing size with time. For Ringo, the radius of gas-phase formamide stays rather constant at around $\sim 2.5$ au. Indeed, Ringo has a relatively constant orbital radius as a function time, 

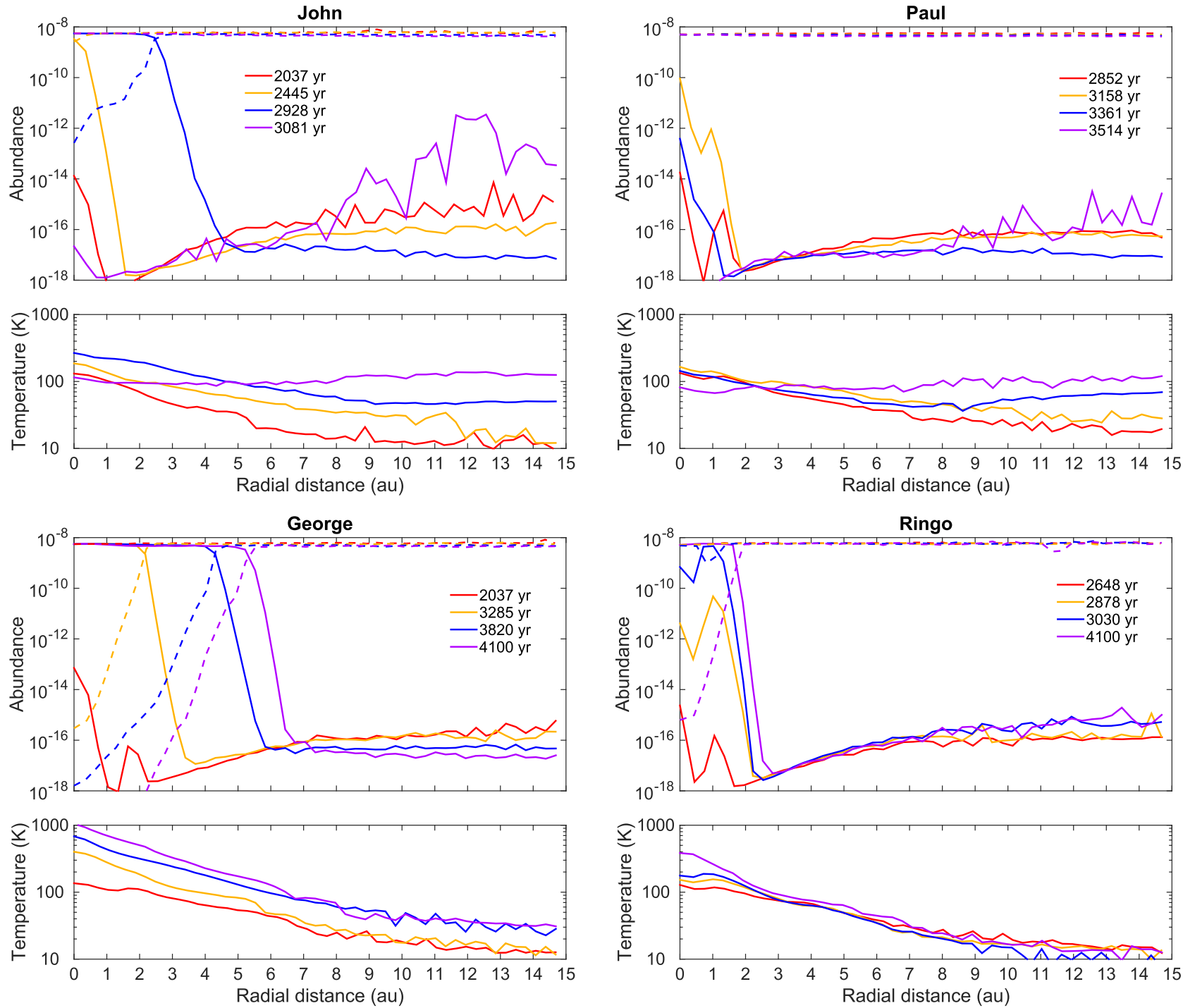

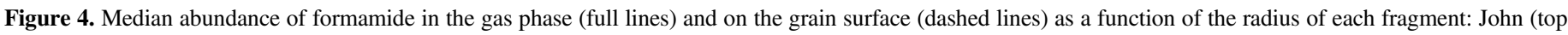

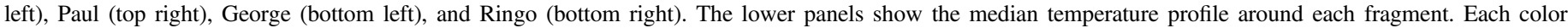
represents a different time, comprised between the formation and destruction time (if any) of each fragment.

thus its radial temperature profile remains the same with time $(T \geqslant 100 \mathrm{~K}$ for $R \lesssim 2.5 \mathrm{au})$. The temperature in Paul is much lower than that of the other fragments $(150 \mathrm{~K}$ versus $300-1100 \mathrm{~K}$, at most), hence the majority of formamide remains frozen onto dust grains. Indeed the gas-phase abundance in Paul is less than a few $10^{-10}$ within $0.5 \mathrm{au}$, corresponding to $<1 \%$ of the total formamide abundance of $10^{-8}$ available on the grains.

\section{Discussion}

\subsection{Comparison with Cometary Abundances of Formamide}

In Section 3.1, we found that formamide is fully frozen on the surface of dust grains soon after the start of the simulation in the outermost regions of the disk and outside the fragments, as a result of their low temperatures and high densities $\left(T \leqslant 10 \mathrm{~K}\right.$ and $\left.n\left(\mathrm{H}_{2}\right) \geqslant 10^{10} \mathrm{~cm}^{-3}\right)$. The solid-phase abundance of formamide in these regions therefore does not change significantly during the remaining evolution of the disk. In a similar way, comets are thought to be formed from pristine material at the very edge of protoplanetary disks that do not undergo significant chemical processing. Therefore, the solid-phase abundance of formamide in our model at timescales $\sim 850$ years from the start of the disk simulation should be representative of such a situation. In order to test this, we gathered observationally determined abundances of formamide and related species for three long-period cometsC/1995 "Hale-Bopp," C/2012 F6 "Lemmon" and C/2013 R1 "Lovejoy" (see Bockelée-Morvan et al. 2000; Biver et al. 2014; Le Roy et al. 2015, and references therein). Table 2 shows these abundances (relative to $\mathrm{H}_{2} \mathrm{O}$ ) alongside abundances measured in the outer disk regions early in the disk simulation. We find that these abundances agree to within a factor of 5 for all species, and note an excellent agreement between the modeled and observed values of formamide. As such, our results show that even massive, unstable disks that undergo gravitational fragmentation can possess cold outer regions within which the abundance of complex organics can be similar to those observed in long-period comets in the solar system. 
Table 2

Solid-phase (Ice) Abundances in the Outer Parts of the Disk Model Compared to Observed Abundances in Long-period Comets

\begin{tabular}{lcc}
\hline \hline Species & Model (\%) & Observed $^{\text {a }}(\%)$ \\
\hline $\mathrm{NH}_{2} \mathrm{CHO}$ & 0.02 & $0.01-0.021$ \\
$\mathrm{NH}_{3}$ & 1.08 & $0.61-0.7$ \\
$\mathrm{H}_{2} \mathrm{CO}$ & 0.23 & $0.7-1.1$ \\
$\mathrm{CO}$ & 9.65 & $4.0-23.0$ \\
$\mathrm{CO}_{2}$ & 27.4 & 6 \\
$\mathrm{CH}_{4}$ & 1.65 & $0.6-0.67$ \\
$\mathrm{CH}_{3} \mathrm{OH}$ & 1.00 & $1.6-2.6$ \\
$\mathrm{HCN}$ & 0.766 & $0.14-0.25$ \\
\hline
\end{tabular}

Notes. All values are relative to water (in percent).

${ }^{a}$ Long-period comets: C/1995 O1 (Hale-Bopp), C/2012 F6 (Lemmon) and C/2013 R1 (Lovejoy). The values are taken from Biver et al. (2014), BockeléeMorvan et al. (2000), and Le Roy et al. (2015; and references therein).

\subsection{Formamide Preservation around "Cold" Fragments}

In Section 3.2, we show that most of the formamide content in Paul $(\sim 99 \%)$ remains on the grain surface during its existence. The "cold" environment around Paul is a direct result of a smaller size and mass $(\sim 2$ au in radius and mass of 3.7 $M_{\text {Jup }}$ ) compared to the other three fragments (with radii between 2.5 and 7 au and masses between 5.4 and $10.3 M_{\text {Jup }}$ ). Paul does not survive long enough to further contract and heat up its surroundings $(T \leqslant 150 \mathrm{~K})$, so water and many other molecules remain on icy grain surfaces. As proposed by Ilee et al. (2017), this effect may have important consequences for the formation of protoplanetary objects if the timescale for dust settling within the fragments is small enough. Indeed, Ilee et al. (2017) inferred that the timescale for centimeter-sized silicate grains to settle to the center of the fragment would be 1000-2000 yr, which is within the $\sim 2000 \mathrm{yr}$ of existence of Paul. Such dust settling might even occur more quickly, either due to icy grains possessing a high sticking co-efficient than bare grains (e.g., Ehrenfreund et al. 2003; Wang et al. 2005), or via hydrodynamic instabilities within the fragments themselves (Nayakshin 2018). Both effects would allow a higher proportion of solid material to sediment toward the center of the fragment. If rocky protoplanetary cores are formed within these regions, then formamide (and other grain species, including larger complex organics) would be incorporated into them. Such rocky cores may go on to become terrestrial-like planets through e.g., tidal downsizing (Nayakshin 2017; Forgan et al. 2018), sequestering primordial complex organics within them.

\subsection{Formamide Preservation around "Hot" Fragments}

As shown in Section 3.2, formamide is thermally desorbed into the gas phase around hot fragments due to high temperatures. In the innermost regions of the fragments where $T \geqslant 500 \mathrm{~K}$, formamide is destroyed via gas-phase reactions for timescales longer than those considered in these simulations. While at larger radii, the temperature drops to levels at which formamide can be retained on dust grains (Figure 4). Thus, while forming protoplanetary fragments would be devoid of solid-phase species, the circumfragmentary material around them would not be. Here, we discuss the content of this circumfragmentary material, and the potential makeup of any planetary satellites that may go on to form from it.

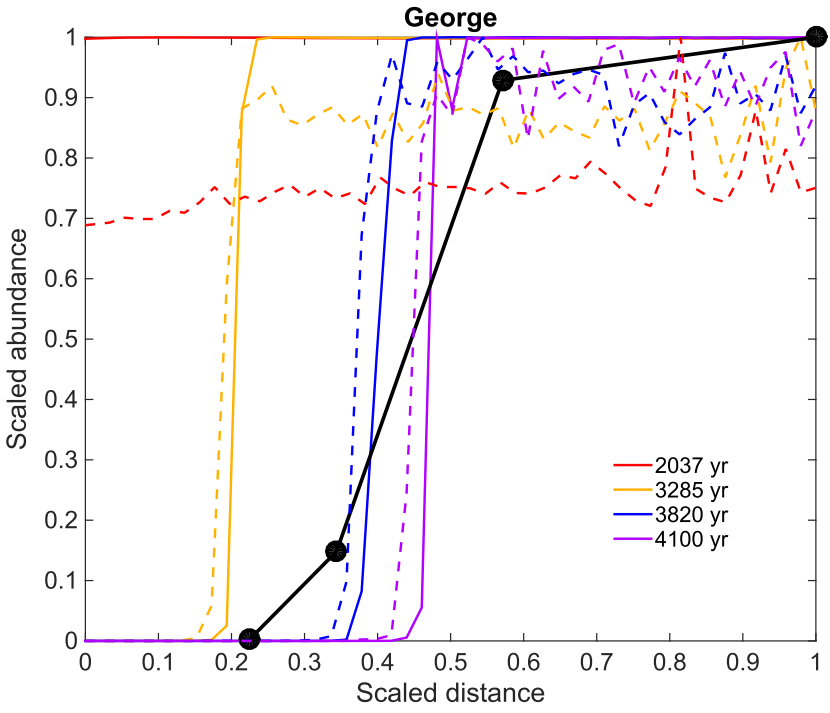

Figure 5. Scaled water (full colored lines) and formamide (dashed colored lines) abundances on the grain surface as a function of the radius of George. The scaled distance used for George is equal to $12 \mathrm{au}$. Each color represents a different time. The black line show the water content inside the four Galilean moons, as a function of their distance from Jupiter (scaled to the distance of Callisto).

In Figure 5, we present the distribution of water (in solid lines) and formamide (see dashed lines) in solid phase around George, the hottest surviving fragment in our simulations (different colors correspond to different timescales). We also overplot the solid water content measured in the Galilean moons: 46-48 wt\% [percentage by mass] for Ganymede, 49-55 wt\% for Callisto, 0\% for Io, and 6-9 wt\% for Europa (Kuskov \& Kronrod 2001, 2005). The $x$-axis has been scaled to the radius of Callisto and to the radius for which the temperature around George equals the temperature of the disk (i.e., at a radius of $12 \mathrm{au}$ ). From Figure 5, we find that the fraction of solid water and formamide in our simulations increases with radius in a similar fashion to that observed for the solid water content in the Galilean moons. Indeed, the low temperatures $(\sim 40 \mathrm{~K})$ and high water abundances found at the outer radius around George are consistent with an icy moon like Callisto being formed at low temperatures, which could have prevented any melting of the ice (while Io, Europa, and Ganymede are completely differentiated bodies, Callisto presents a mantle without any separation between the ice and the rock material; Kuskov \& Kronrod 2005). Therefore, formamide could also be retained in satellites formed around protoplanetary fragments from gravitationally unstable disks.

We stress that this comparison is valid regardless of the formation scenario proposed for a Jupiter-like system (e.g., whether the planet is formed via core accretion or gravitational instability; Canup \& Ward 2002; Mosqueira \& Estrada 2003), because the expected shapes of the radial temperature profile around nascent protoplanets formed via either mechanism are the same (see Cleeves et al. 2015; Szulágyi et al. 2017). As such, water and formamide will always follow a similar radial behavior as shown in Figure 5. Thus, while significant chemical processing can still occur in the later, Class II stages of protoplanetary disk evolution, our results demonstrate that such processing is not required to match the radial abundance profiles of solar system satellites, and similarities can be in place at early times for objects formed via gravitational fragmentation of the disk. 


\section{Summary}

We have studied the formation and evolution of the key prebiotic molecule formamide in the context of a young, massive circumstellar disk that gravitationally fragments into bound protoplanets. We find that formamide is located in the gas phase in the innermost $(r<15 \mathrm{au})$ regions of the disk, and in three of the four fragments. It remains on the grain surfaces in the cold, outer regions of the disk where its abundance is consistent with that observed in long-period comets. In one fragment, formamide remains on dust grains long enough to allow sedimentation, implying that rocky protoplanetary cores formed via, e.g., tidal downsizing, can inherit primordial prebiotic material directly from the protostellar nebula. In the circumfragmentary material, the radial abundance of solid formamide and water is consistent with the behavior of solid water observed across Galilean satellites.

In combination, our results show that when calculated explicitly, the chemical composition of protoplanets and protosatellites formed in gravitationally unstable disks can be similar to those expected from core accretion, and can even be consistent with the composition of objects within our own solar system.

The authors are grateful to Serena Viti, Richard Nelson and Craig Agnor for valuable discussions, and to the anonymous referee for suggestions that helped to improve the manuscript. D.Q. acknowledges the support received from the STFC through an Ernest Rutherford Grant (grant No. ST/M004139). J.D.I. acknowledges support from the DISCSIM project, grant agreement 341137 under ERC-2013-ADG, and support from the STFC (grant No. ST/R000549/1). I.J.-S. acknowledges partial support by the MINECO and FEDER funding under grants ESP2015-65597-C4-1 and ESP2017-86582-C4-1-R. D.H.F. acknowledges support from the ECOGAL project, grant agreement 291227, under ERC-2011-ADG. C.H. acknowledges that this project has received funding from the European Research Council (ERC) under the European Union's Horizon 2020 research and innovation program (grant agreement No 681601). C.H. is a Winton Fellow and this research has been supported by Winton Philanthropies.

Software: sphNG (Bate et al. 1995); UCLCHEM (Holdship et al. 2017).

\section{ORCID iDs}

David Quénard (1) https://orcid.org/0000-0002-2969-3985

John D. Ilee (1) https://orcid.org/0000-0003-1008-1142

Izaskun Jiménez-Serra (ii) https://orcid.org/0000-00034493-8714
Duncan H. Forgan (1) https://orcid.org/0000-0003-1175-4388

Cassandra Hall (1) https://orcid.org/0000-0002-8138-0425

Ken Rice (1) https://orcid.org/0000-0002-8138-0425

\section{References}

Altwegg, K., Balsiger, H., Berthelier, J. J., et al. 2017, MNRAS, 469, S130 Awad, Z., Viti, S., Collings, M. P., \& Williams, D. A. 2010, MNRAS, 407, 2511

Bate, M. R., Bonnell, I. A., \& Price, N. M. 1995, MNRAS, 277, 362

Biver, N., Bockelée-Morvan, D., Debout, V., et al. 2014, A\&A, 566, L5

Bockelée-Morvan, D., Lis, D. C., Wink, J. E., et al. 2000, A\&A, 353, 1101

Canup, R. M., \& Ward, W. R. 2002, AJ, 124, 3404

Caselli, P., Keto, E., Bergin, E. A., et al. 2012, ApJL, 759, L37

Cleeves, L. I., Bergin, E. A., \& Harries, T. J. 2015, ApJ, 807, 2

Drozdovskaya, M. N., Walsh, C., van Dishoeck, E. F., et al. 2016, MNRAS, 462, 977

Ehrenfreund, P., Fraser, H. J., Blum, J., et al. 2003, P\&SS, 51, 473

Evans, M. G., Ilee, J. D., Boley, A. C., et al. 2015, MNRAS, 453, 1147

Forgan, D., Rice, K., Stamatellos, D., \& Whitworth, A. 2009, MNRAS, 394, 882

Forgan, D. H., Hall, C., Meru, F., \& Rice, W. K. M. 2018, MNRAS, 474, 5036

Furuya, K., Drozdovskaya, M. N., Visser, R., et al. 2017, A\&A, 599, A40

Goesmann, F., Rosenbauer, H., Bredehöft, J. H., et al. 2015, Sci, 349, doi:10. 1126/science.aab0689

Hall, C., Forgan, D., \& Rice, K. 2017, MNRAS, 470, 2517

Hasegawa, T. I., Herbst, E., \& Leung, C. M. 1992, ApJS, 82, 167

Holdship, J., Viti, S., Jiménez-Serra, I., Makrymallis, A., \& Priestley, F. 2017, AJ, 154, 38

Ilee, J. D., Boley, A. C., Caselli, P., et al. 2011, MNRAS, 417, 2950

Ilee, J. D., Forgan, D. H., Evans, M. G., et al. 2017, MNRAS, 472, 189

Kahane, C., Ceccarelli, C., Faure, A., \& Caux, E. 2013, ApJL, 763, L38

Kuskov, O. L., \& Kronrod, V. A. 2001, Icar, 151, 204

Kuskov, O. L., \& Kronrod, V. A. 2005, SoSyR, 39, 283

Le Roy, L., Altwegg, K., Balsiger, H., et al. 2015, A\&A, 583, A1

McElroy, D., Walsh, C., Markwick, A. J., et al. 2013, A\&A, 550, A36

Minissale, M., Dulieu, F., Cazaux, S., \& Hocuk, S. 2016, A\&A, 585, A24

Mosqueira, I., \& Estrada, P. R. 2003, Icar, 163, 198

Nayakshin, S. 2017, PASA, 34, e002

Nayakshin, S. 2018, arXiv:1808.05151

Öberg, K. I., Guzmán, V. V., Furuya, K., et al. 2015, Natur, 520, 198

Quénard, D., Jiménez-Serra, I., Viti, S., Holdship, J., \& Coutens, A. 2018, MNRAS, 474, 2796

Rawlings, J. M. C., Hartquist, T. W., Menten, K. M., \& Williams, D. A. 1992, MNRAS, 255, 471

Saladino, R., Crestini, C., Pino, S., Costanzo, G., \& Di Mauro, E. 2012, PhLRv, 9, 84

Szulágyi, J., Mayer, L., \& Quinn, T. 2017, MNRAS, 464, 3158

Visser, R., Doty, S. D., \& van Dishoeck, E. F. 2011, A\&A, 534, A132

Viti, S., Collings, M. P., Dever, J. W., McCoustra, M. R. S., \& Williams, D. A. 2004, MNRAS, 354, 1141

Wakelam, V., Loison, J.-C., Mereau, R., \& Ruaud, M. 2017, MolAs, 6, 22

Walsh, C., Loomis, R. A., Öberg, K. I., et al. 2016, ApJL, 823, L10

Walsh, C., Millar, T. J., Nomura, H., et al. 2014, A\&A, 563, A33

Wang, H., Bell, R. C., Iedema, M. J., Tsekouras, A. A., \& Cowin, J. P. 2005 , ApJ, 620, 1027

Yoneda, H., Tsukamoto, Y., Furuya, K., \& Aikawa, Y. 2016, ApJ, 833, 105 